\title{
Cognitive Study of Image Schema and Dying-mind in Tagore's Near-Death Experience (NDE) Poems
}

\author{
Mohammad Shalauddin', Mohammed Shamsul Hoque ${ }^{2}$ \& Touhid Bhuiyan ${ }^{3}$ \\ ${ }^{1}$ Human Resource Development Institute (HRDI), Daffodil International University \\ (DIU),Bangladesh. Email: salauddin@hrdinstitute.org \\ ${ }^{2}$ Department of English, Daffodil International University (DIU), Bangladesh. \\ ${ }^{3}$ Department of Software Engineering (SWE), Daffodil International University (DIU), \\ Bangladesh.
}

Received May 31, 2017; Revised July 10, 2017; Accepted July 15, 2017; Published August 18, 2017.

\begin{abstract}
This study aims at analyzing image schemas (Johnson, 1987) and stages of grief (Kubler-Ross, 1969) in Tagore's near-death experience (NDE) poems written in the last ninety days before his death. Selected verses of the period in question are analyzed using benchmarks set by Johnson and Kubler-Ross. Relation between the schemas and some linguistic forms, themes and moods of these poems are also analyzed. Findings include Dying-mind is centered to self and influences the type and frequency of all the schemas within it. Similar mental stages of grief-Denial, Anger, Bargaining, Depression and Acceptance - are also found in the NDE poems. Though death is depicted by Tagore even to the stature of "Shyam" in his earlier poems, it is presented as "A moving screen of varied fears" in the end, which is supported by the theory of Kubler-Ross.
\end{abstract}

Keywords: Cognitive Linguistics, Image Schema, Kubler-Ross model, NDE poems, Tagore.

\section{Introduction}

Cognitive linguistics started to be studied seriously in the late 1970s as a new branch of cognitive science and has been increasingly referenced since the 1980s (Croft, 2004). Emerged at the periphery of philosophy, psychology, anthropology, linguistics, artificial intelligence, and neuroscience, cognitive science is an interdisciplinary scientific study of the mind and intelligence (Thagard, 2005, p. ix). Cognitive science primarily focuses on to explain how people accomplish the various kinds of thinking process like perception, problem solving, attention, emotion, memory, reasoning etc. (Thagard, 2005). It covers a range of complementary, overlapping, and competing theories (Evans, 2006, p. 3). It also has important political and social implications (Gardner, 1985, p. 359).

According to Croft and Cruse (2004), cognitive linguistic approach to language works on explaining the relationship between language and its cognitive processing in the human brain guiding through three major hypotheses. First, rejection of an autonomous cognitive unit in the mind; second, grammar is understood in terms of conceptualization; and third, it believes that "knowledge of language emerges from language use" (Croft, 2004, p. 1). Taylor (2006) describes language as a symbolic system, which mirrors through patterns, namely syntax, semantics and

(C) AesthetixMS 2016. This Open Access article is published under a Creative Commons Attribution Non-Commercial 4.0 International License (http://creativecommons.org/licenses/by-nc/4.0/), which permits non-commercial re-use, distribution, and reproduction in any medium, provided the original work is properly cited. For citation use the DOI. For commercial re-use, please contact editor@rupkatha.com. 
morphology and, hence, inherently meaningful. In this regard, metaphor and image schema are important as they convey bodily experiences and our mind generates some kinds of mental pictures on the basis of bodily or sensory interaction with the world (Johnson, 1987). This picture uses some basic templates or frames like Container, Path, and Force etc. to understand the events that occur in everyday life (Stockwell, 2005, 2006). In the field of computational linguistic research this are the metaphorical intuitive pattern for tangible human-computer interaction (HCI) system (Hurtienne, 2007).

Psycholinguistics is also an interdisciplinary, empirical and theoretical field of research which deals with mental process and linguistics (Altmann, 2001). According to Dry (1992), though research in psycholinguistics works with text but it is not the core issue like form, grammar, rhythm, metaphor etc. but it is the text-receiver's perceptual process and visual theories offered by linguistics (Dry, 1992, p. 437).

This study, taking all these developments in the field of cognitive linguistics and psycholinguistics, aims to explore if relevant poems present such schemas and near-death psychological reactions have been dealt with by the greatest of poets in Bengali literature, Rabindranath Tagore (1861-1941) in his poems immediately before his death.

\section{Rationale}

Generally, researchers including Latha (2013), Rao (2015), Cattoi (2015) over many decades have viewed Tagore as embracing death with open arms as "Shyam', a loving spiritual companion. But there has always been a recurrent undercurrent in the Tagore psyche, which amounts to a kind of pangs and sufferings expressed through images of darkness and fear in his poems. Incidentally, this side of the Tagore Psyche, or, in other words, Tagore's emotional reaction to advent of death, has been untapped until now. This study aims to unfold it in the light of established theories on psychology of death and dying.

\section{Methodology}

This study is mainly descriptive-analytic. It explores five near-death experience (NDE) poems by Rabindranath Tagore (1861-1941) written in the last ninety days prior to his death. It reviews cognitive schema and psychological trauma in the poems, "On the bank of the Rupa-narayana" (রূপ-নারানের কূনে), “On your birthday, at the festival of gifts" (তব জন্ম দিবসের দানের উৎসবে), “The sun of the first day" (প্রথম দিনের সূর্য) , "Sorrow's dark night again and again” (দুঃখের আঁধার রাত্রি বারে বারে) and "You have strewn the path of your creation" (তোমার সৃষ্টির পথ রেখেছ আকীর্ণ করি). A literature review was carried on death and dying poetry, image schema by Johnson (1987) and five stages of grief by Kubler-Ross (1969) to record how they are prescribed and interpreted by different researchers/scholars. Because, this study considers 90 days as near-death state, a selection of five poems by Tagore composed close to the time of his demise has been analyzed. After careful selection, a thorough investigation and analysis of the selected poems in Bangla and their translation in English was carried on to validate the existence of the schemas and grief stages in the poems. Coupled with qualitative analysis, quantitative data analysis has been carried with respect to schema type, number and frequency in the light of Johnson's (1987) theory and five stages of grief advocated by Kubler-Ross (1969). Results are presented based on the most frequent types occurring in the poems down to the least frequent types. In the next, with 
respect to schema found relationship between linguistics form and theme of the poem has been done. Finally, a cross-model mapping between two theories - Image schema by Johnson (1987) and grief stages by Kubler-Ross (1969) - were put side by side to match the grief stages and image schemas in the selected poems which represent the inside view of the dying-mind. Alongside, description of the psychological stages of Tagore during the period in questions, his mood reflection in the poems, especially, of those connected to the image schemas, are discussed and analyzed to find how Tagore has dealt with the NDE (near-death experience) at the last phase of his life.

\section{Death and Dying Poetry}

Numerous poets have written poems motivated and/or, influenced by death and its grieving process. The death poem is a distinct type or genre of poetry that reflects the death, both in general and in particular for the author. Unlike, elegy (lamenting) and eulogy (in praise of the dead), poems that talk about death of someone else in different form and theme (Abrams, 1999), dying or near-death experience (NDE) poems are considered NDE poems of a particular poet (web. 1). In Japanese culture best known as "death poem" often prepared years in advance before death (Smith, 2014). We have selected such poems of Tagore, which he wrote in the last three months of his life. These poems project his own near-death experience. The end of life or dying poems describes the most difficult and painful emotions and feelings associated with death of an author. Tagore is no exception.

There are considerable numbers of famous poets in the world literature who have been influenced by advent of death in their own life, which contributed to their creative contribution to literature through poetry on themes of death depicting their own thoughts, feelings and emotion. Examples of famous death poems include "The Dream" by Lord Byron (1788-1824), "The Dance of Death" by Charles Baudelaire (1821-1867), "Because I could not stop for Death" by Emily Dickinson (1830-1886), "Letter in November" by Sylvia Plath (1932-1963) etc.

Kubler-Ross (1969) is the pioneer on analysis of near-death experience (NDE). She developed a model to analyze NDE of individuals in general. Though the Kubler-Ross Model is the most prominent and accepted model of grief, many others have proposed similar models. They are, Engel (1964); Bowlby \& Parkes (1970); Worden (1982); Sanders (1992) and Rando (1993) (Hango, 2015, p. 8-9). Kubler-Ross and others, while developing their models, carried study on dying and the frails in their terminal stage, mainly from psychological point of view. In particular, Kubler-Ross carried on her experiment and used 13 poems of Tagore in her narrative of the final report on the research. All the 13 Tagore poems depict death experience. Hence, the current research aims to further analyze the last five poems written in the last 90 days before Tagore's demise which we considered as NDE poems of Tagore.

\section{Image Schemas}

According to Johnson (1987) "an image schema is recurring dynamic pattern of our perceptual interaction and motor programs that gives coherence and structure to our experience." (1987, p. xiv, xvi). These patterns are the mental pictures originated through the physical experience (embodied) of meaningful comprehension and reasoning. Here experience is understood in a very rich and broad sense that includes: "basic perceptual, motor-program, emotional, historical, social and linguistics dimensions." (Johnson, 1987, p. xvi). Each schema consists of a small number of objects and relations, structured through by the virtue of perceptions, images, and events. It is a 
two-dimensional mental representation which operates at a layer of human thought process where abstract proposition structure works on one side, and particular concrete images, on the other. (Johnson, 1987, p. 29).

Johnson provided a long list of basic image schemas in the book, "Body in the Mind" (1987, p. 126), Three of these basic schemas are as follows:

\subsection{Container Schema}

According to Johnson (1987), the Container schemas relate to the experience of being in a place enclosed by a boundary like a room, a bed, a box etc. The Container has three basic structural elements - an inside, a boundary and an exterior. It is a kind of space where elements reside or move either inside or outside it. From the experiential grounding as Johnson cited by ISCAT, "there are typical schemata for physical containment and containment is transitive and if $B$ is in A, then whatever is in B is also in A" (web. 2). Example of Container in English: "What obligations have you gotten yourself into? I can't get out of it, I'm locked into it.” (Lakoff, 1991, p. 33).

\subsection{Path Schema}

A Path schema presents the experience of motion in a direction. It has three basic construction elements. They are: a source or starting point, an end-point or a goal, and a sequence connecting the source with the goal (Johnson, 1987, p. 113). Motion experiences like Journey, up/down, front/back, over/under, into/out of (web. 2). Example of Path in English: "Tom has gone a long way toward changing his personality." (Johnson, 1987).

\section{$5 \cdot 3$ Force Schema}

Force schema presents the experience of causal interaction. It includes a sequence of causation, a direction and intensity of the force and, a path of a motion of the source and a target of the source (Johnson, 1987). Force usually implies the exertion of physical strength in one or more directions. Force dynamics can be shortly characterized as "a stronger force opposing a weaker force head on, with all-or-none conditions" Talmy (1988, p. 98). Example of Force in English: "He can exert his influence on her. Reagan brought pressure to bear upon his cabinet." [Lakoff, 1991, p. 131].

\section{Kubler-Ross Model (1969): Five Stages of Grief}

Near about half a century ago, Swiss-born psychiatrist and advocate Elisabeth Kubler-Ross in her 1969 book, "On Death and Dying", presented the five stage of grief, academically famed as KublerRoss model. The stages of grief postulate how we react to the news that we have a terminal illness (Zizek, 2016). It is the near-death experience (NDE) that all dying experiences. According to the model, our response evolves over five stages and they are: Denial, Anger, Bargaining, Depression and Acceptance. These are the series of emotional experiences that the terminally ill patients go through prior to death. They are popularly known by the acronym DABDA (Denial, Anger, Bargaining, Depression and Acceptance).

Through interviewing more than 400 dying patients in four years this "certain generalizations" has been stated (1972, p. 179). Most, but not all, patients pass through the five states and they necessarily don't come in the same order (1972, p. 179). These are a collection of common experiences that most dying faces. Kubler-Ross later applied these stages to any form of catastrophic personal loss, such as, death of a loved one, divorce, drug addiction, joblessness, chronic illness, and even minor losses. Despite few academicians, both sufferers and therapists have reported the usefulness of the model in a wide variety of situation. Lim (2013) has found 
similar feedback from the consumers who experienced grief as a result of a let-down in anticipation of the iPhone 5. Musician like Hango (2015) has composed brass ensemble based on five stages of grief and very recently, philosopher Slavoj Zizek (2016) has found a similar combination of desperate reactions to the refugees from Africa and Middle East to the Western Europe.

Kubler-Ross's book, "On Death and Dying” (1969), "is not meant to be a textbook on how to manage dying patients, nor is it intended as a complete study of the psychology of the dying" (Kubler-Ross, 1969 , p. x). It is simply an account of a new and a challenging attempt of reverseclassroom-dialogue experiment between the doctor and the patient (Kubler-Ross being the student, patient/dying as the teacher). Refocusing on the patient as a "human being" and as a "teacher", it is the learning class room from the patient about the final stages of life with its three symptoms: anxieties, fears, and hopes. This resulted in a meaningful experience manifesto for us who do not know their own finality. From pure experiential ground, Kubler-Ross hoped that few who can engage with those near-death dialogues will discover a mutually gratifying experience emerged from the experience of the dialogue with the dying. Along with that, they will learn about the deep functionality of the human mind, the unique human aspects of existence and be prepared for their own finality with fewer anxieties. Kubler-Ross's learning came to the realization that the fear triggered by "hopelessness", "helplessness" and "isolation" is the only crisis that a dying faces but the death itself (1969, p. 240). Kubler-Ross (1972) summarizes, "psychiatrically it is extremely important to appreciate that, in terms of the unconscious, we cannot conceive of our own death and that, in addition, we cannot differentiate between the wish and the deed.....we live today in the illusion that, since we have mastered so many things, we shall be able to master death too." (1972, p. 179).

By this she attributed a new argument that the society has been changed to a "DeathDenying Society" in which people attempt to deny that they die. An Internet-based End of Life Care Education Program at Lurie Comprehensive Cancer Center also came up with similar conclusive findings like: "For most of us, the inevitability of our own death can be denied, repressed, or otherwise not considered" (web. 3).

Followings are the brief descriptions of the stages that Kubler-Ross described in her book “On Death and Dying” (1969):

\subsection{Denial}

Denial is usually a temporary defense and a simple refusal to accept the fact. Most reacts at first with the statement: "No, not me, it cannot be true" (Kubler-Ross, 1969) and soon be replaced by partial acceptance. In Tagore's voice picked by Kubler-Ross: "Man barricades against himself." (Tagore, Stray Birds, 79, 1916).

\subsection{Anger}

When the first stage of denial can no longer deny the fact, it is replaced by feelings of anger, rage, envy, and resentment. The logical next question becomes "Why me?" (Kubler-Ross, 1969). In Tagore's voice picked by Kubler-Ross: "We read the world wrong and say that it deceives us." (Tagore, Stray Birds, 75, 1916).

\subsection{Bargaining}

The third stage, bargaining is an attempt to postpone or diminish the fact. Most bargains are made with God and are usually kept in secret. To postpone the inevitable happening negotiation takes place like: "If God has decided to take us from this earth and he did not respond to my angry 
pleas, he may be more favorable if I ask nicely" (Kubler-Ross, 1969). In Tagore's voice picked by Kubler-Ross: "The wood cutter's axe begged for its handle from the tree./The tree gave it." (Tagore, Stray Birds, 71, 1916).

\subsection{Depression}

At this state, the dying person begins to understand the certainty of their death. The individual may become silent and spend much of their time mourning. Two types of depression they face: one is reactive depression and the second is preparatory depression (Kubler-Ross, 1969). First one is different in nature and the second one is more common to undergo in order to prepare themselves for their final separation from this world. Statement may include: "I'm going to die, so why bother with anything?" (Zizek, 2016). In Tagore's voice cited by Kubler-Ross: “The world rushes on over the strings of the lingering heart making the music of sadness." (Tagore, Stray Birds, 44, 1916).

\subsection{Acceptance}

In this last stage, individuals embrace the inevitable loss. It is not a happy stage. It is almost a stage void of feelings. It is as if the pain had gone, the struggle is over, and there comes a time for "the final rest before the long journey" (Kubler-Ross, 1969) as one patient phrased. The likely sense in this stage is: "what's the use" or "I just cannot fight it any longer" (Kubler-Ross, 1969). In Tagore's voice mentioned by Kubler-Ross: "I have got my leave. Bid me farewell, my brothers! I bow to you all and take my departure...A summons has come and I am ready for my journey." (Tagore, Gitanjali, 93, 1913).

\section{Results}

\subsection{Tagore's Near-Death Experience of NDE Poetry}

Tagore wrote five poems in the last 90 days/three months' time frame are shown in following Table 1.

Table 1. Tagore's near-death experience or NDE poetry

\begin{tabular}{|c|c|c|c|}
\hline & NDE Poems & $\begin{array}{c}\text { Days } \\
\text { before } \\
\text { death }\end{array}$ & $\begin{array}{c}\text { Verses } \\
\text { (Bengali) }\end{array}$ \\
\hline 1 & On the bank of the Rupa-narayana (রূপ-নারানের কূলে) & 85 & 15 \\
\hline 2 & On your birthday, at the festival of gifts (তব জন্মদিবলের দানের উৎসবে) & 24 & 16 \\
\hline 3 & The sun of the first day (প্রথম দিনের সূর্য) & 10 & 11 \\
\hline 4 & Sorrow's dark night again and again (দুঃখের আঁধার রাত্রি বারে বারে) & 8 & 12 \\
\hline 5 & You have strewn the path of your creation (তোমার সৃষ্টির পথ রেখেছ আকীর্ণ করি) & 7 & 25 \\
\hline
\end{tabular}

As shown in table 1, they were written consecutively $85,24,10,8$ and 7 days before his death. In Bengali version poem 1 contains 15 verses, 2 contains 16 verses, 3 contains 11 verses, 4 contains 12 verses and 5 contains 25 verses, all together 52 sentences (separated by full stop in Bengali) and total 293 words. All the above poems are popularly known as "Shesh Lekha" (The last writings) (web. 4). 


\subsection{Types and Frequency of Image Schemas in Selected Five poems of Tagore}

The schemas found in the five poems are categorized in to three groups, which match with the types introduced by Johnson (1987). They are shown in the following table 2.

Table 2. Types and frequency of schemas in five poems

\begin{tabular}{|c|c|c|c|c|c|c|c|c|c|c|}
\hline & \multirow{2}{*}{\multicolumn{2}{|c|}{$\begin{array}{c}1 \\
\text { On the bank of } \\
\text { the Rupa- } \\
\text { narayana }\end{array}$}} & \multirow{2}{*}{\multicolumn{2}{|c|}{$\begin{array}{c}\mathbf{2} \\
\text { On your } \\
\text { birthday, at the } \\
\text { festival of gifts }\end{array}$}} & \multirow{2}{*}{\multicolumn{2}{|c|}{$\begin{array}{l}\frac{3}{\text { The sun of the }} \\
\text { first day }\end{array}$}} & \multirow{2}{*}{\multicolumn{2}{|c|}{$\begin{array}{c}4 \\
\begin{array}{c}\text { Sorrow's dark } \\
\text { night again and } \\
\text { again }\end{array}\end{array}$}} & \multirow{2}{*}{\multicolumn{2}{|c|}{$\begin{array}{c}5 \\
\text { You have } \\
\text { strewn the path } \\
\text { of your creation }\end{array}$}} \\
\hline & & & & & & & & & & \\
\hline & No. & $\%$ & No. & $\%$ & No. & $\%$ & No. & $\%$ & No. & $\%$ \\
\hline Container & 9 & $90 \%$ & 5 & $71 \%$ & 7 & $88 \%$ & 5 & $45 \%$ & 11 & $69 \%$ \\
\hline Path & 1 & $10 \%$ & 2 & $29 \%$ & 1 & $12 \%$ & 5 & $45 \%$ & 4 & $25 \%$ \\
\hline Force & o & $0.0 \%$ & o & $0.0 \%$ & o & $0.0 \%$ & 1 & $10 \%$ & 1 & $6 \%$ \\
\hline Total: & 10 & $100 \%$ & 7 & $100 \%$ & 8 & $100 \%$ & 11 & $100 \%$ & 16 & $100 \%$ \\
\hline
\end{tabular}

As shown in table 2, the three types of schemas found in the poems are Container, Path and Force schema. Container schema is more frequent than the other two in all the poems. Only poems 4 and 5 contain all three schemas: Container, Path and Force. Poem 4 and poem 5 contain 45\% and 69\% Container schema, 45\% and 25\% Path schema and $10 \%$ and 6\% Force schema respectively. Poems 1, 2 and 3 contain Container and Path schema of which Container schema $90 \%, 71 \%, 88 \%$ in poems 1,2 and 3 respectively. And Path schema in poems 1,2 and 3 contain 10\%, $29 \%, 12 \%$ consecutively in these three poems. Some examples are as follows:

Poem 1 verse 1: “রূপ-নারানের কূলে/জেগে উঠিলাম;/জানিলাম এ জগৎ/স্বপ্ন নয়।” (Appendix- A)

"On the bank of the Rupa-narayana/I woke up,/I knew that this world/Is not a dream." (Appendix- B)

In the above verse, two Container schemas are used: (1) 'on the bank of the Rupanarayana' ('রূপ-নারানের কূনে') is a physical place that denotes Container where poet woke, and (2) the 'world' ('জগৎ') is the Container where poet realized this world in no dream.

Path schema is linked with verb containing the concept of motion. Example is as follows:

Poem 2 verse 4: “দাতা আর গ্রহীতার যে সংগম লাগি/বিধাতার নিত্যই আগ্রহ/আজি তা সার্থক হল,/বিশ্বকবি তাহারি বিস্ময়ে/তোমারে করেন আশীর্বাদ-/তাঁর কবিত্বের তুমি সাক্ষীরূপে দিয়েছ দর্শন/বৃষ্টিধৌত শ্রাবনের/নির্মল আকাশে।"

(Appendix-A)

"At the insistence of the destiny,/the donor and the donee are together today./Wondering, the world poet blesses you./In his poetry, you are implied/From the rain-washed sky of July."

\section{(Appendix- B)}

In the above verse, two Path schemas and one Container schema are used: (1) 'donor' ('দাতা') and 'donee' ('গ্রহীতা') is two separate entity linked: 'together' ('সংগম') with the interest of divine which denotes the Path schema, (2) 'poet' ('কবি') and 'you' ('তোমারে') is two separate entity linked with the virtue of blessing indicates Path schema, and (3) 'sky' ('আকাশ') is the Container schema where the 'his' (‘তাঁর’) and 'you' ('তুমি’) reside. 
Force schema is linked with verb containing the concept of power accompanied by two nouns. Example is as follows:

Poem 4 verse 1: “দুঃখের আঁধার রাত্রি বারে বারে/এসেছে আমার দ্বারে;/একমাত্র অস্ত্র তার দেখেছিনু/কষ্টের বিকৃত ভান, ত্রাসের বিকট ভঙ্গি যত-/অন্ধকারে ছলনার ভূমিকা তাহার।" (Appendix- A)

"Sorrow's dark night, again and again,/ has come to my door; its only weapon I saw,/ was pain's twisted brow, fear's hideous gestures/ precluding its deception in darkness." (Appendix- B)

In the above verse, three Container and one Force schemas are used: (1) 'my' ('আমার') denotes the Container and 'door' ('দ্বার') is the physical object within the self-container where grief darkness came again and again, (2) 'I saw' ('দেখেছিনু') is the Container and 'weapon' ('অস্ত্র') is the physical object within the self-container, (3) 'fear' ('ত্রাসের') indicates the Force that is hideous gesture, and (4) 'in darkness' ('অন্ধকারে’) denotes Container where 'its' ('তাহার’) role is deceptive.

\subsection{Grief Stages and Order in Selected Five Poems}

Grief stages and order found in the five poems introduced by Kubler-Ross (1969) are shown in the following figure 1.

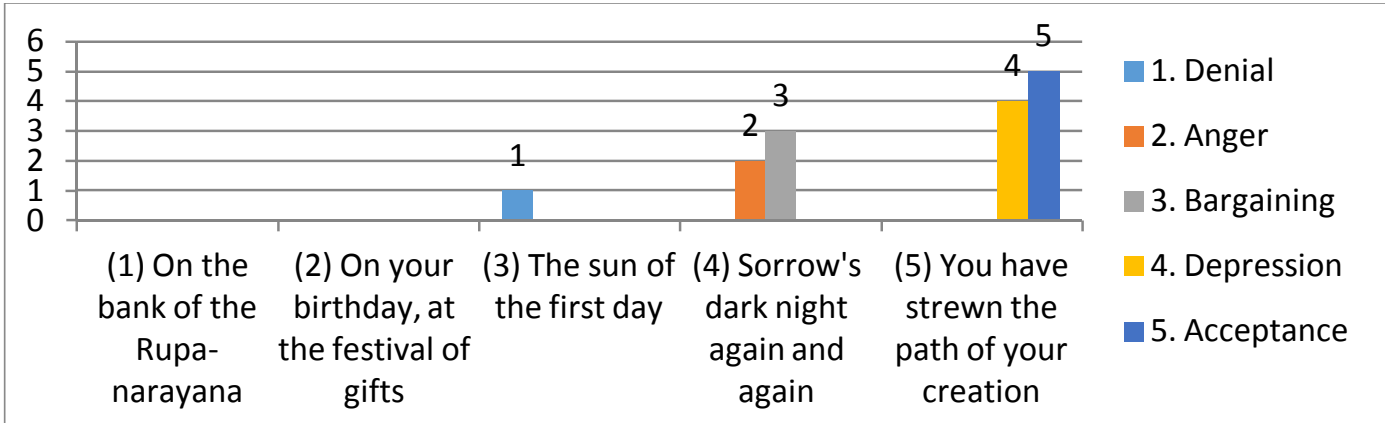

Figure 1. Grief stages and order found in five poems

As shown in figure 1, all these five stages are clear and ordered starting from the $3^{\text {rd }}$ poem written 10 days before his death and ending to the $5^{\text {th }}$ poems written 7 days prior to his death in the selection under study. Poem 3, "The sun of the first day", written 10 days before his death contains Denial stage and overall mood is discourse rejection in the form of question-answer. It produces image and sense of temporary defense through a denial argument made by the dyingmind. Examples is as follows:

Poem 3 verse 1 and 2: “প্রথম দিনের সূর্য/প্রশ্ন করেছিল/সত্তার নূতন আবির্ভাবে-/কে তুমি?/মেলে নি উত্তর।/বৎসর বৎসর চলে গেল।/দিবলের শেষ সূর্য/শেষ প্রশ্ন উচ্চারিল/পশ্চিম সাগর তীরে/নিস্তব্ধ সন্ধ্যায়-/কে তুমি?/পেল না উত্তর।"(Appendix-

"The sun of the first day/ had asked/at the new advent of the being: who are you?/No answer came.

Year after year passed,/the last sun of the day/uttered the last question on the shore/ of the West Sea,/by a mute evening: who are you?/Received no answer." (Appendix- B)

Poem 4, "Sorrow's dark night again and again", written 8 days before his death contains Anger and Bargaining stages and overall mood is argumental. Verse 1 and 2 produces image and 
sense of anger through a betraying dispute and verse 3 and 4 produces image and sense of bargaining through a defeating argument made by the dying-mind. Examples are as follows:

Poem 4 verse 1 and 2: “দুঃখের আঁধার রাত্রি বারে বারে/এসেছে আমার দ্বারে;/একমাত্র অস্ত্র তার দেখেছিনু/কষ্টের বিকৃত ভান, ত্রাসের বিকট ভঙ্গি যত-/অন্ধকারে ছলনার ভূমিকা তাহার।/যতবার ভয়ের মুখোশ তার করেছি বিশ্বাস/ততবার হয়েছে অনর্থ পরাজয়।" (Appendix-A)

"Sorrow's dark night, again and again,/has come to my door; its only weapon I saw,/was pain's twisted brow, fear's hideous gestures/precluding its deception in darkness. Whenever I have believed in its mask of dread/fruitless defeat has followed. (Appendix-B)

Poem 4 verse 3 and 4: “এই হার-জিত খেলা, জীবনের মিথ্যা এ কুহক,/শিশ্ডকাল হতে বিজড়িত পদে পদে এই বিভীষিকা/দুঃখের পরিহানে ভরা।/ভয়ের বিচিত্র চলচ্ছবি-/মৃত্যুর নিপুণ শিল্প বিকীর্ণ আঁধারে ।” (Appendix- A)

"This game of defeat and victory is life's delusion:/From childhood, at each step, clings this specter-filled with sorrow's mockery. A moving screen of varied fears-/death's skilful handiwork wrought in scattered gloom." (Appendix- B)

The last poem 5, "You have strewn the path of your creation", contains Depression and Acceptance stages and overall mood is calm and submission meaning acceptance. Verse 1 produces image and sense of depression through a dampen argument and verse 2 and 3 produces image and sense of acceptance through an acceptance argument made by the dying-mind. Examples are as follows:

Poem 5 verse 1: “তোমার সৃষ্টির পথ রেখেছ আকীর্ণ করি/বিচিত্র ছলনাজালে/হে ছলনাময়ী!” (Appendix- A) "You have strewn the path of your creation/with a varied deceit,/O deceitful Dame." (AppendixB)

Poem 5 verse 2 and 3: “মিথ্যা বিশ্বাসের ফাঁদ পেতেছ নিপুণ হাতে/সরল জীবনে!/এই প্রবঞ্চনা দিয়ে মহত্ত্বেরে করেছ চিহ্তিত;/তার তরে রাখ নি গোপন রাত্রি।" (Appendix- A)

"A crafty hand has set the trap/ of vain confidence/in a life without guile. Even you have stigmatised greatness/with that fraud,/without keeping the night a secret for him." (Appendix- B)

It is noted that, though Kubler-Ross (1969) postulates that death is not the problem for the dying but the fear of death (1969, p. 240). In poem 5, despite fear of his own death, a glorified hopes for a trophy winner notably found in the dying-mind of Tagore. This may be linked to the nobility of being a poet. Following figure 2, topographic mood-map illustrates more clear view of mental reaction stages and chronological mood transmissions at the final stage. 


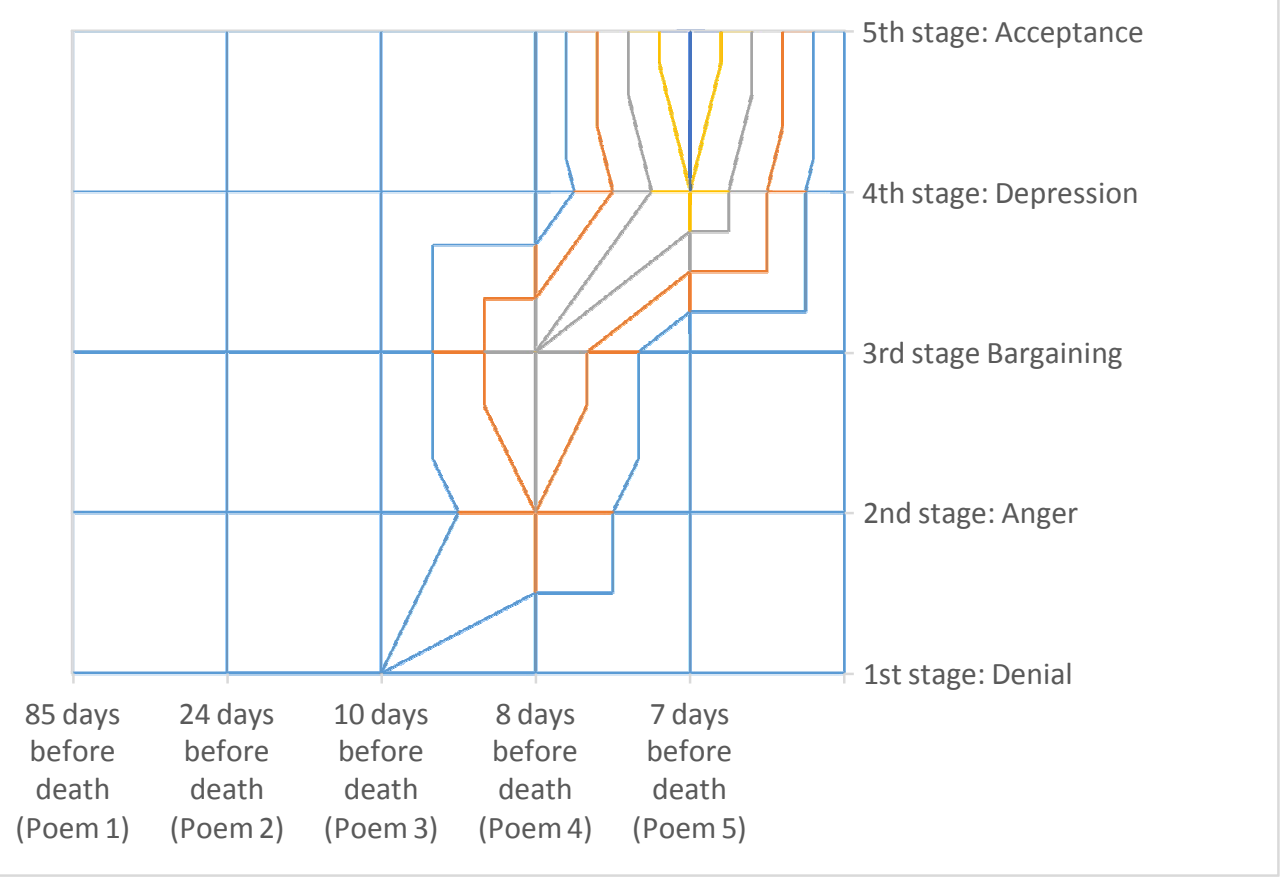

Figure 2. Topographic mood-map of Tagore's dying-mind

As shown in figure 2, Denial stage started 10 days before his death and rest grief stages Anger, Bargaining, Depression and Acceptance gradually occurred in the poems following the order set by Kubler-Ross Model (1969).

\subsection{Linguistic Forms and Schema Relations}

Based on the data collected, Container schema found in the poems all the way and has clear link with some special linguistic forms. It usually comes in the form of a noun phrase or suffix phrase with the final letter of the noun e.g., ‘রূপ-নারানের কূলে/জেগে উঠিলাম’, ('On the bank of the Rupnarayan/I woke') in poem 1 presents a Container in the form of a suffix phrases: ‘-এর’ (of/in), as in 'নারানের’ (নারান+এর) and '-এ’ (on), as in ‘কূলে’ (কূল+এ), with the final letter of each noun phrases: ‘রূপ-নারান’ ('Rupnarayan') and ‘কূল’ ('bank'); and ‘নিস্তব্ধ সন্ধ্যায়’ ('By a mute evening') in poem 2, presents a Container in the form of a inflexion phrase: '- $য ়$ ' (in) with the final letter of the noun 'সন্ধ্যা' ('evening').

Path schema aslo is found in the poems all the way in the form of verb dynamics. Verbs containing the concept of motion, or the semantic feature of movement in a direction, or nouns linked with conjunctional phrase derived from such verb or semantic motion feature e.g., 'ভয়ের বিচিত্র চলচ্ছবি_' ('A moving screen of varied fears-') in poem 4 'চলচ্ছবি' ('moving screen'), presents a Path schema indicating motion to forward direction and the noun: 'ভয়' ('fears') derives from this dynamicity; 'শেষ পুরস্কার নিয়ে যায় লে যে/আপন ভাণ্ডারে’ ('carrying his final trophy/he returns to his store-house.') in poem 5, presents a Path schema in the form of a feature of physical movement: 'নিয়ে যায়' ('returns to'/out of) form one place to another place: ‘ভাণ্ডারে’ ('store-house'); and ‘দাতা আর গ্রহীতার যে সংগম লাগি/বিধাতার নিত্যই আগ্রহ/আজি তা সার্থক হল’, ('At the insistence of the destiny,/the donor and the donee are together 
today.') in poem 5, presents a Path schema in the form of two nouns: ‘দাতা' ('donor') and ‘গ্রহীতা’ ('donee') linked with a conjunctional phrase: ‘দাতা আর গ্রহীতার’ (‘donor and the donee’).

For the Force schema, the linguistic form association is less restricted, and occuring between nouns derived from verb or dynamic feature; one serving as the obstacle while the other one having the power to or trying to force the other, e.g., 'ত্রাসের বিকট ভঙ্গি যত-' ('fear's hideous gestures') in poem 4, 'ত্রাসের' ('of fear/fear's') indicates the Force schema that is fear imposes and affects; and 'এই প্রবঞ্চনা দিয়ে মহত্ত্বেরে করেছ চিহ্নিত' ('Even you have stigmatized greatness/with that fraud') in poem 5, ‘চিহ্নিত' ('stigmatized') presents Force schema by establishing link between two nouns derived from the verb with a implying force.

\subsection{Poem Themes and Schema Relation}

Reader's perceptual view on the theme of the poem 1, "On the bank of the Rupa-narayana" is selfrealization. The poet tries to see inside of himself, his own image empowered with realization of the truth. The theme of the poem 2, "On your birthday, at the festival of gifts" is self-declaration as the poet by making use of birthday celebration party of other. The poem was written on the birth anniversary of Nandita Devi (granddaughter of Tagore, daughter of Mira Devi). The theme of the $3^{\text {rd }}$ poem, "The sun of the first day", tells about the self-inquiring through the question "Who are you?" and answering to it. "The sun of the first day" ('প্রথম দিনের সূর্য') refers to birth and "the last sun of the day" ('দিবসের শেষ সূর্য') refers to death. Birth and Death in the poem have been depicted through the simile of Sunrise and Sunset. The theme of the $4^{\text {th }}$ poem, "Sorrow's dark night again and again" is a self-death view of a motion picture full of horror. And the theme of the poem 5, "You have strewn the path of your creation" is a path creation with a varied deceit and a dignified hope for a final trophy winner. The "You", the deceitful dame, in the poem is stigmatized by the nature and acquires the right to peace though people call him a victim of many deceptions that the creator brings. Based on table 2, the image schemas found in relation to the themes of the five poems are shown in the following table 3 .

Table 3. Poem themes and schema relation

\begin{tabular}{|c|c|c|c|}
\hline \multirow[t]{2}{*}{ Poem Theme } & \multicolumn{3}{|c|}{ Schemas } \\
\hline & Container & Path & Force \\
\hline Poem 1 : Self-realization & 9 & 1 & o \\
\hline Poem 2 : Self-declaration as poet & 5 & 2 & o \\
\hline Poem 3 : Self-inquiry : who are you? & 7 & 1 & o \\
\hline Poem 4 : Self-death view and fear & 5 & 5 & 1 \\
\hline Poem 5 : Deceit path and dignified hope & 11 & 4 & 1 \\
\hline Total & 37 & 13 & 2 \\
\hline
\end{tabular}

As shown in table 3, poem 1 contains 9 Container, 1 Path and o Force schemas. Poem 2 contains 5 Container, 2 Path and o Force schemas. Poem 3 contains 7 Container, 1 Path and o Force schemas. Poem 4 contains 5 Container, 5 Path and 1 Force schemas. And last poem 5 contains 11 Container, 4 Path and 1 Force schemas. The total number of Container schemas in all five poems is 37 compared 13 Path schemas and only 2 Force schemas. So, it may by conclude that the themes of the poems have got a closer connection with Container schema.

The theme of darkness is often likened to death in many poetic creations including paintings by many modern artists. Tagore is no exception to this. The later paintings of Tagore 
have been depicted with "an overpowering sense of darkness and the grotesque" (Sen, 2010, p.39) which is reflected in his NDE poems as well.

Although Cattoi and Moreman (2015) like many others including Choudhery and Shrivastava (2015), Sankar et al, (2016) etc. goes along with the traditional appreciation of the theme of acceptance of death in Tagore's early poems, there is evidence of fear of death in the imagery of "darkness and the grotesque" (Sen, 2010) in his paintings as well as in the poem like "Moron re, Tuhu momo shyamsoman" where the grotesque darkness appears vividly in the beginning of the poem.

\subsection{Dying-Mind and Schema Relation}

This study aims at discovering the image schema and dying mental stages in Tagore's final poems. The research finding proves that dying-mind mood is centered to self, and, thus influences the type and frequency of the image schemas within it as shown in the above table 3. It is evident in Tagore's poetry written in the last three month of his life, Container schema (68\%) which is more frequent than the Path schema (18\%) and Force schema (14\%) where overall perceptual interaction and mental imaging process are centered round self-container. The following figure 3 illustrates the whole scenario.

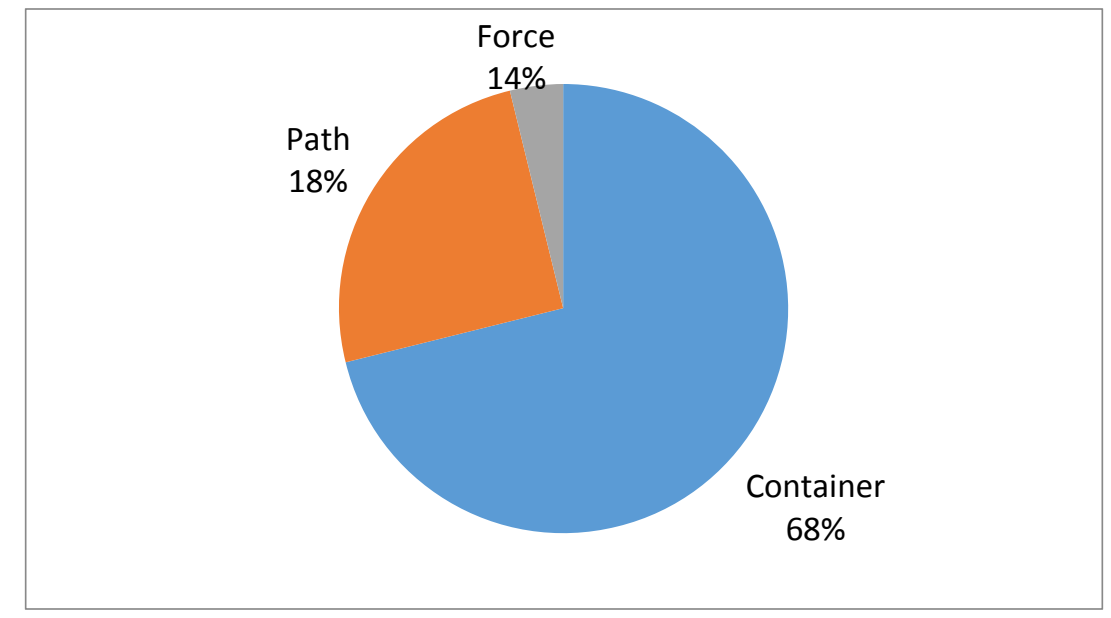

Figure 3. Dying-mind and schema relation

\subsection{Rabindranath Tagore in "On Death and Dying"}

"On Death and Dying" by Kubler-Ross (1969) contains 12 chapters. Each chapter starts with Tagore's verses containing a presentation of death as depicted in the cited extract of the chosen poems. Interestingly Kubler-Ross uses Tagore's verse to start each of the chapters except the $12^{\text {th }}$ chapter where Kubler-Ross uses Tagore's verse to conclude the chapter as well. From Tagore's four English poem books, Kubler-Ross have selected 8 verses from "Stray Birds" (1916), 3 verses from "Gitanjali" (1912), 1 verse from "Fruit Gathering" (1916) and 1 verse from "The Fugitive" (1921). As the book ends with Tagore's verse: "The small truth has words that are clear;/ the great truth has great silence." (Stray Birds, 176, 1916). Tagore has no mention at all in the entire book of Kubler-Ross but the verses. Putting Tagore's verses in the head of each chapter and at the start and end of the $12^{\text {th }}$, Kubler-Ross made Tagore as the 'silent head teacher' to her death and dying experiment. 


\section{Discussion and Related Work}

A similar approach to that presented here can be found in English language poem of William Wordsworth edited by Miall (1997) and in Persian language poem of poet Omar Khayyam edited by Esfehani (2012), poet Sohrab Sepehri edited by Sharifi (2012), poet Hafez edited by Asgari (2013) and poet Fereydoon Moshiri edited by Salehnejad (2015). Sharifi (2012) and Salehnejad (2015) found similar results that prove the dynamicity in human life that influences the image schema within it. The poems they worked on are "The Footsteps of Water" and "The Passenger" by Sohrab Sepehri and "The Lane" by Fereydoon Moshiri. Form the poem title we can sense the notion of movement and distance. Thus, Path schema is more frequent than Container schema in their study. On the other hand, in this study since, death is the resistance of imminent moving experience of life; Container schema is more frequent than the Path schema. In both studies, Force schema is least frequent.

\section{Conclusion}

Image Schema is a unique component of complex human mental process, specially, while experiencing the trauma of the advent of death in the final stage of life. Based on the data collected and analyzed in this paper, it can be concluded that dying-mind gets cocooned within itself and so influences the type and frequency of the image schemas. It is evident from this investigation that Container schema $(68 \%)$ is more frequent than the Path schema (18\%) and Force schema $(14 \%)$ where overall perceptual interaction and mental imaging process are centered round self-container. Tagore's poems written in the last three months of his life also illustrate the same overpowering "sense of darkness and the grotesque" (Sen, 2010) depicted in his later paintings. Similar NDE effects are experienced in the form of traumatic warnings leading to death and this happened in order of the stages advocated by Kubler-Ross (1969). In addition, a dignified hope stage has been found in dying-mind of Tagore that resembles the magnanimity of the poet. Putting Tagore's verses in the head of each chapter and at the start and end of the $12^{\text {th }}$ chapter of the book, "On Death and Dying" (1969), Kubler-Ross put Tagore to the position of the 'silent head teacher' to her death and dying experiment. It is also evident that some linguistic forms of Bangla are connected with the image schemas, such as, Container schema is linked with nouns and suffix phrases with the final letter of the noun; Path schema is linked with verb containing the concept of motion and Force schema is linked with verb containing the concept of power accompanied by two nouns. Though death is depicted in Tagore's poetry as a fearless companion, a spiritual lover, in his early poems but in the end, Tagore depicts death as "A moving screen of varied fear." This is manifest in the NDE poems written by Tagore in the last 90 days before his death.

\section{References}

Abrams, M. H., (1999). A Glossary of Literary Terms. Seventh Edition, 1999, ISBN: 0-15-505452-X, Library of Congress Catalog Card Number: 98-7257.

Altmann, G. T. M., (2001). The language machine: Psycholinguistics in review. British Journal of Psychology, 92(1), 129-170.

Asgari, T., (2013). The Study of Image Schemas in Hafez Poems: Cognitive Perspective, International Journal of Language and Linguistics. Vol. 1, No. 4, 2013, pp. 182-19o. doi: 10.11648/j.ijll.20130104.23 
Cattoi, T., \& Moreman, C. M., (2015). Death, Dying, and Mysticism: The Ecstasy of the End, First published in 2015 by Palgrave Macmillan, ISBN: 978-1-137-47207-6.

Croft, W., Cruse, D. A., (2004). Cognitive Linguistics. Cambridge University Press, Jan 22, 2004.

Choudhery, P. \& Shrivastava, S., (2015). Theme of Death in Rabindranath Tagore's Poetry, New Man International Journal of Multidisciplinary Studies, ISSN: 2348-1390, Vol. 2, Issue 6, June 2015, pp. 27-30.

Dry, H. A., (1992). Foregrounding: An Assessment. Language in Context: Essays for Robert E. Longacre.

Esfehani, L. S. (2012). Cognitive poetics as a literary theory for analyzing Khayyam's poetry. Procedia-Social and Behavioral Sciences, 32, 314-320. http://dx.doi.org/10.1016/j.sbspro.2012.01.046

Evans, V., Green, M. (2006). Cognitive Linguistics and introduction. Edinburgh University Press. ISBN o 748618317.

Gardner, H., (1985). The Mind's New Science. A History of the Cognitive Revolution. New York: Basic Books, 1985, ISBN 0-465-04634-7.

Hango, C. R., (2015). The Five Stages of Grief: A Composition for Brass Ensemble, 2015.

Hurtienne, J., \& Israel, J. H. (2007). Image schemas and their metaphorical extensions: intuitive patterns for tangible interaction. In Proceedings of the ist international conference on Tangible and embedded interaction (pp. 127-134). ACM.

Johnson, M. (1987). The Body in the mind: The bodily basis of meaning, imagination, and reason. Chicago: University of Chicago Press.

Kubler-Ross, E., (1969). On death and dying. Routledge, ISBN 0-415-04015-9.

Kubler-Ross, E., Wessler, S., \& Avioli, L. V. (1972). On death and dying. JAMA, 221(2), 174-179.

Lakoff, G., \& Johnson, M. (1980). Metaphor we live by. Chicago: University of Chicago Press.

Lakoff, G., Espenson, J., and Schwartz, A., (1991). Master Metaphor List. Second Edition, Cognitive Linguistics Group, University of California at Berkeley, October, 1991.

Latha, T., (2013). Theme of Love E Death in Tagore's Gitanjali, International Journal of English and Education, ISSN: 2278-4012, Volume: 2, Issue: 1, January 2013, 247-252.

Lim, W. M. (2013). Revisiting Kubler-Ross's Five Stages of Grief: Some Comments on the iPhone 5. Journal of Social Science 9 (1): 11-13, 2013.

Miall, D. S. (1997). The body in literature: Mark Johnson, metaphor, and feeling. Journal of Literary Semantics, 26(3), 191-210.

Rao, V., (2015). Rabindranath Tagore: Gitanjali, Research Journal of English Language and Literature (RJELAL), Vol.3.3.2015 (July-Sep), 2395-2636 (print): 2321-3108 (online), 350-36o.

Salehnejad, L., \& Naghizadeh, M. (2015). Cognitive Study of Image Schemas in a Poem by "Fereydoon Moshiri'. International Journal of English and Education, ISSN: 2278-4012, Vol. 4, Iss. 4.

Sankar, G., Sriprabha, M., Kumar, S. S., \& Kumar, R. S. (2016). Theme of Adore and Nationalism in Tagore's Gitanjali: A Study. Higher Education of Social Science, 11(2), 15-17. doi: http://dx.doi.org/10.3968/8812

Sen, A., (2010). "Beyond Borders": Rabindranath Tagore's Paintings and Visva-Bharati, Rupkatha Journal on Interdisciplinary Studies in Humanities, Volume 2, Number 1, Special Issue, Visual Arts. http://rupkatha.com/V2/n1/Rabindranathtagorepaintings.pdf

Sharifi, S., \& Mayamei, N. (2012). Cognitive study of schema in two poems by "Sohrab Sepehri". ProcediaSocial and Behavioral Sciences, 32, 329-333. http://dx.doi.org/10.1016/j.sbspro.2012.01.048

Smith, H., (2014). The Stuff of Dreams: Kawanabe Kyōsai's Nirvana Painting of Matsuura Takeshirō. Impressions, no. 35 (Spring 2014), pp. 96-135. 
Stockwell, P., (2005). Cognitive poetics: An introduction. Routledge.

Stockwell, P., (2006). Schema Theory: Stylistic Applications. In: Keith Brown, (Editorin-Chief) Encyclopedia of Language \& Linguistics, Second Edition, volume 11, pp. 8-13. Oxford: Elsevier.

Talmy, L. (1988). Force dynamics in language and cognition. Cognitive science, 12(1), 49-100. http://onlinelibrary.wiley.com/doi/10.1207/s15516709cog1201_2/pdf

Taylor, J. R., (2006). Cognitive Semantics, Encyclopedia of Language \& Linguistics (2006), vol.2, Oxford: Elsevier, pp. 569-582.

Thagard, P. (2005). Mind: Introduction to cognitive science. Second Edition. Cambridge, MA: MIT press. ISBN-81-203-3003-X.

Web. 1, NDE Poetry. http://nderf.org/NDERF/Articles/poetry.htm. (June 2016).

Web. 2, Image Schemata Catalogue, ISCAT. http://iscat.stefciu.de. (June 2016).

Web. 3, EndLink. Resource for End of Life Care Education. http://endlink.lurie.northwestern.edu. (June 2016).

Web. 4, Shesh Lekha. https://archive.org/details/SheshLekha1941 (June 2016).

Zizek, S., (2016). Against The Double Blackmail: Refugees Terror and Other Troubles, Penguin Random House UK. ISBN: 978-0-241-27884-0.

\section{Appendix- A}

(১)

রূপ-নারানের কূলে

জেগে উঠিলাম;

জানিলাম এ জগৎ

স্বপ্ন নয়।

রক্তের অক্ষরে দেখিলাম

আপনার রূপ-

চিনিলাম আপনারে

আঘাতে আঘাতে

বেদনায় বেলনায়;

সত্য যে কঠিন,

কঠিনেরে ভালোবাসিলাম-

সে কখনো করে না বঞ্চনা।

আমৃত্যুর দুঃখের তপস্যা এ জীবন-

সত্যের দারুণ মূল্য লাভ করিবারে,

মৃত্যুতে সকল দেনা শোধ ক’রে দিতে।

[উদয়ন। শান্তিনিকেতন। ১৩ মে ১৯৪১। রাত্রি ৩।১৫ মিনিট ।] [উদয়ন। শান্তিনিকেতন। ১৩ জুলাই ১৯৪১। সকাল।]
তব জন্মদিবসের দানের উৎসবে

বিচিত্র সজ্জিত আজি এই

প্রভাতের উদয়-প্রাঙ্গণ।

নবীনের দানসত্র কুসুমে পল্লবে

অজস্র প্রচুর।

প্রকৃতি পরীক্ষা করি দেখে

ক্ষণে ক্ষণে আপন ভাণ্ডার, তোমারে সম্মুখে রাখি পেল সে সুযোগ।

দাতা আর গ্রহীতার যে সংগম লাগি

বিধাতার নিত্যই আগ্রহ

আজি তা সার্থক হল,

বিশ্বকবি তাহারি বিস্ময়ে

তোমারে করেন আশীর্বাদ-

তাঁর কবিত্বের তুমি সাক্ষীরূপে দিয়েছ দর্শন

বৃষ্টিধৌত শ্রাবণের

নির্মল আকাশে।

(2) 
প্রথম দিনের সূর্য

প্রশ্ন করেছিল

সত্তার নূতন আবির্ভাবে-

কে তুমি?

মেলে নি উত্তর।

বৎসর বৎসর চলে গেল।

দিবলের শেষ সূর্য

শেষ প্রশ্ন উচ্চারিল

পশ্চিম সাগর তীরে

নিস্তব্ধ সন্ধ্যায়-

কে তুমি?

পেল না উত্তর।

[জোড়াসাঁকো। কলকাতা। ২৭ জুলাই ১৯৪১। সকাল।]

\section{(8)}

দুঃখের আঁধার রাত্রি বারে বারে

এসেছে আমার দ্বারে;

একমাত্র অস্ত্র তার দেখেছিনু

কষ্টের বিকৃত ভান, ত্রাসের বিকট ভঙ্গি যত-

অন্ধকারে ছলনার ভূমিকা তাহার।

যতবার ভয়ের মুখোশ তার করেছি বিশ্বাস

ততবার হয়েছে অনর্থ পরাজয়।

এই হার-জিত খেলা, জীবনের মিথ্যা এ কুহক,

শিশুকাল হতে বিজড়িত পদে পদে এই বিভীষিকা-

দুঃখের পরিহালে ভরা।

ভয়ের বিচিত্র চলচ্ছবি-

মৃত্যুর নিপুণ শিল্প বিকীর্ণ আঁধারে।

[জোড়াসাঁকো। কলকাতা। ২৯ জুলাই ১৯৪১। বিকাল।]
এই প্রবঞ্চনা দিয়ে মহত্ত্বেরে করেছ চিহ্নিত;

তার তরে রাখ নি গোপন রাত্রি।

তোমার জ্যোতিক্ষ তারে

যে পথ দেখায়

সে যে তার অন্তরের পথ,

সে যে চিরস্বচ্ছ,

সহজ বিশ্বাসে সে যে

করে তারে চিরসমুজ্ব্বল।

বাহিরে কুটিল হোক, অন্তরে লে ঋজু

এই নিয়ে তাহার গৌরব।

লোকে তারে বলে বিড়ম্বিত।

সত্যেরে সে পায়

আপন আলোকে-ধৌত অন্তরে অন্তরে।

কিছুতে পারে না তারে প্রবঞ্চিতে,

শেষ পুরস্কার নিয়ে যায় লে যে

আপন ভাঞ্জারে।

অনায়ালে যে পেরেছে ছলনা সহিতে

লে পায় তোমার হাতে

শান্তির অক্ষয় অধিকার।

[জোড়াসাঁকো। কলকাতা। ৩০ জুলাই ১৯৪১। সকাল ৯।৩০ মিनिট।]

Source: https://archive.org/details/SheshLekha1941 (o5 June 2016).

$(\varangle)$

তোমার সৃষ্টির পথ রেখেছ আকীর্ণ করি

বিচিত্র ছলনাজালে

হে ছলনাময়ী!

মিথ্যা বিশ্বাসের ফাঁদ পেতেছ নিপুণ হাতে

সরল জীবনে! 


\section{Appendix- B}

(1)

On the bank of the Rupa-narayana

I woke up,

I knew that this world

Is not a dream.

I read my own name

In letters of blood,

I recognised myself

Blow after blow,

With pain after pain;

Truth is stern,

I loved the stern

Which never deceives.

This life until death

is an ascetic quest of sorrow,

To earn the terrible price of Truth

Pay off all debt while dying.

Udayan, Santiniketan, 13 May 1941, 3:15 am.

Translated by Prithwindra Mukherjee.

Source:http://www.kaurab.com/english/bengali _poetry/rabindranath3.pdf

\section{(2)}

On your birthday, at the festival of gifts,

The courtyard of this morning is a appropriately dressed.

The festival of giving by the ever-new

Is manifested in abundance in flower

With you as the witness,

The nature examines its treasures every

moment.

At the insistence of the destiny, the donor and the donee are together today.

Wondering, the world poet blesses you.

In his poetry, you are implied

From the rain-washed sky of July.

Udayan, Santiniketan, 13 July 1941, morning.

Online collection. Source:

http://www.monsoonletters.com/genre/sheshlekha

\section{(3)}

The sun of the first day

Had asked

At the new advent of the Being :

Who are you?
No answer came.

Year after year passed.

The last sun of the day

Uttered the last question on the shore

of the Western sea,

By a mute evening:

Who are you?

Received no answer.

Jorashako, Kolkata, 27 July 1941, morning.

Translated by Prithwindra Mukherjee.

Source:http://www.kaurab.com/english/bengali

_poetry/rabindranath3.pdf

(4)

Sorrow's dark night, again and again,

Has come to my door.

Its only weapon I saw,

Was pain's twisted brow, fear's hideous gestures

Preluding its deception in darkness.

Whenever I have believed in its mask of dread

Fruitless defeat has followed.

This game of defeat and victory is life's delusion :

From childhood, at each step, clings this spectre.

Filled with sorrow's mockery.

A moving screen of varied fears-

Death's skilful handiwork wrought in

scattered gloom.

Jorashako, Kolkata, 29 July 1941, afternoon.

Translated by Dr Amiya Chakravarty.

Source:http://gypsyscholar.com/31604historicev entstagoret.html

(5)

You have strewn the path of your creation

With a varied deceit,

O deceitful Dame.

A crafty hand has set the trap

of vain confidence

In a life without guile.

Even You have stigmatised greatness

with that fraud,

Without keeping the night a secret for him.

The path on which Your star guides him

Is indeed his inward path,

Ever frank,

In innate confidence,

He keeps that ever resplendent. 
Without, however it be crooked, it is straight forward in heart, Therein lies his pride, Even though people call him a victim, He is in communion with truth bathed in its own light in the heart of heart. Nothing can deceive him, Carrying his final trophy He returns to his store-house.
He who has effortlessly suffered all deceit Acquires from Your hand The right to Peace everlastingly. Jorashako, Kolkata, 30 July 1941, 9:30 am. Translated by Prithwindra Mukherjee. Source:http://www.kaurab.com/english/bengali _poetry/rabindranath3.pdf

Mohammad Shalauddin is working as Researcher at Human Resource Development Institute (HRDI) of Daffodil International University (DIU). He is the author of a book and doing intense research on Bangla computational linguistics, especially poetic text. His research area is cognitive science, cognitive poetics, computational linguistics, psycholinguistics etc. He is currently working on a corpus research project at DIU.

Mohammed Shamsul Hoque is working as Associate Professor at Department is English, Daffodil International University (DIU).

Touhid Bhuiyan is working as Professor and Head at Department of Software Engineering, Daffodil International University (DIU). 